\title{
High-Intensity Small-Sided Games versus Repeated Sprint Training in Junior Soccer Players
}

\author{
by \\ Niyazi Eniseler ${ }^{1}$, Çă̆atay Şahan ${ }^{1}$, Ilker Özcan ${ }^{1}$, Kıvanç Dinler ${ }^{1}$
}

The aim of this study was to compare the effects of high-intensity small-sided games training (SSGT) versus repeated-sprint training (RST) on repeated-sprint ability (RSA), soccer specific endurance performance and short passing ability among junior soccer players. The junior soccer players were recruited from of a professional team (age $16.9 \pm 1.1$ years). The tests included the repeated-shuttle-sprint ability test (RSSAT), Yo-Yo Intermittent Recovery Test level 1 (Yo-Yo IR1) and Loughborough Soccer Passing Test (LSPT). Nineteen participants were randomly assigned to either the small-sided games training (SSGTG) $(n=10)$ or repeated-sprint training group $(R S T G)(n=9)$. Small-sided games or repeated-sprint training were added to the regular training sessions for two days of the regular practice week. The Wilcoxon signed-rank and Mann-Whitney $U$ tests were used to examine differences in groups and training effects. A time $x$ training group effect was found in the improvement of short-passing ability for the smallsided games training group which showed significantly better scores than the repeated-sprint training group $(p \leq 0.05)$. Both groups showed similar improvements in RSAdecrement $(p<0.05)$. Only the repeated-sprint training group improved in the Yo-Yo IR1 $(p<0.05)$. This study clearly shows that high-intensity small-sided games training can be used as an effective training mode to enhance both repeated sprint ability and short-passing ability.

Key words: repeated-sprint ability, small-sided games, soccer specific endurance, passing ability, repeated-sprint training.

\section{Introduction}

Competing within the elite level of sports, professional soccer players are expected to possess well-developed physical capabilities complementing the technical and tactical demands of contemporary soccer (Aziz et al., 2000). Among these important technical abilities, short passing ability is considered one of the utmost importance. In various studies analyzing World Cups, the importance of passing and short passing on goals scored is emphasized (Hughes and Franks, 2005; Sajadi and Rahnama, 2007). In addition to the technical abilities, physical capabilities such as superior aerobic capacity (Chamari et al., 2005), muscular strength, power (Wisloff, 2004), repeated sprint ability (Bishop et al., 2011; Spencer et al., 2005) and soccer specific endurance (Bangsbo, 1994) are also important. Soccer-specific endurance, as measured using the Yo-Yo Intermittent Recovery Tests, has been found to be related to the amount of highintensity activity completed during the match (Krustrup et al., 2003). Yo-Yo Intermittent Recovery Tests are sensitive to training interventions and important in discriminating between top-class and moderate ability soccer players (Bangsbo et al., 2012).

Game analysis studies have demonstrated that soccer requires participants to repeatedly produce maximal or near maximal actions of short duration with brief recovery periods (Spencer et al., 2005). The ability to repeat these high-intensity and short duration efforts following short

1 - Department of Physical Education and Sports, Celal Bayar University, Şehzadeler, 45010, Manisa, Turkey. 
recovery periods has been termed as 'the repeated sprint ability' and has been shown to be a good predictor of game-related physical performance in top-level professional soccer players (Rampinini et al., 2007). In soccer players, significant correlations have been reported between both very high-speed running and sprinting distance during actual match play and mean sprint times in RSA tests (Rampinini et al., 2009). Similarly to Yo-Yo tests, RSA tests have also shown to discriminate professionals from amateur players (Aziz et al., 2008; Impellizzeri et al., 2008; Reilly et al., 2000).

Repeated-sprint training appears to be an effective mode of multicomponent training and is known to improve maximal oxygen uptake and repeated-sprint ability (Bishop et al., 2011). Soccer training should commonly include physical exercises aimed to enhance both aerobic fitness and repeated-sprint ability (Bravo et al., 2008). Consequently, the optimal design and implementation of training strategies that enhance RSA of soccer players are of significant interest to soccer coaches and players. Because of the various metabolic (ie., oxidative capacity, PCr recovery and $\mathrm{H}+$ buffering), neuromuscular (ie., muscle activation and recruitment strategies) and mechanical determinants of RSA, various training strategies may impact this ability (Spencer et al., 2005). However, there has been little research in order to find the best training method to improve RSA. Both speed and/or endurance-oriented training programs are used to develop RSA of team sport players. Three principal training methods have emerged: repeated-sprint training (Fernandez et al., 2012) or sprint interval training (Gibala et al., 2006), high-intensity aerobic training (interval training) (Bravo et al., 2008; Edge et al., 2005) and lastly, aerobic-oriented small-sided games training (Buchheit et al., 2009; Hill-Haas et al., 2009; Jensen et al., 2007; Katis and Kellis, 2009). Results of all these studies are encouraging since all methods have shown to improve RSA significantly from 1.5 to 3\% (Buchheit et al., 2010).

While RST and interval training may be an alternative means for improving player's RSA, other important soccer components, such as technical and tactical skills, perceptual skills, visual scanning and decision making are neglected. Small-sided games are generally considered a preferred training method, since many coaches believe that this training allows for the concurrent development of physical, technical, tactical and decision-making abilities in soccer players (Serpell et al., 2011). This sport-specific training method can be used for creating an effective competitive environment while improving RSA. Thus, the inclusion of smallsided games as part of soccer training is quite common in soccer clubs at all levels (Reilly and Gilbourne, 2003). Training studies focusing on the development of RSA in team sport players have mostly investigated the impact of dissociated training approaches (Buchheit et al., 2009; Bravo et al., 2008). However, a congested schedule, as often found among elite soccer clubs, often makes it problematic for coaches attempting to simultaneously integrate these different training components.

Only few studies have compared the effects of generic interval or RS training versus high intensity small-sided games training strategies on RSA and soccer specific endurance performance (Bravo et al., 2008; Hill-Haas et al., 2009; Impellizzeri et al., 2006; Reilly and White, 2005). To the authors' knowledge, no studies have compared the effects of SSG versus RS training on both RSA and technical skills in soccer players. Thus, there is a paucity of data pertaining to the effects of SSG and RS training on technical skills. Therefore, the main purpose of this study was to measure the effects of high-intensity small-sided game training vs RS training on RSA, soccer specific endurance performance (Yo-Yo IR1) and passing skills performances (LSPT) by including a 6-week intervention to regular training sessions of junior male soccer players from a professional team during the pre- and regular season. It was hypothesized that both SSG and RS training intervention would be equally effective at improving RSAdecrement and RSAmeantime, RSAbesttime and soccer specific endurance performance, but only SSGT intervention would induce enhanced short-passing ability.

\section{Methods}

The study was designed to compare the effects of SSG and RS training on junior male soccer players' technical and fitness variables during specific tests. A parallel 2-group, randomized, controlled, longitudinal (pre-test and 
post-test) design was employed.

Before and after the intervention period, anthropometrical assessments, the Yo-Yo IR1 and RSSA tests were used to measure the players' physical capacities and the LSPT was used to evaluate short-passing abilities. The experimental design is presented in Table 1. All of the tests were performed in the morning and were preceded by a day of rest. The players refrained from high-intensity exercise for 24 hours before the testing sessions. During the testing period, the players were asked to consume their usual meal at least 3 hours before the scheduled testing time.

\section{Participants}

Junior soccer players were recruited from a professional team that competed at the national level. The mean $( \pm \mathrm{SD})$ physical characteristics of the soccer players were: age, $16.9 \pm 1.1$ years; body mass, $65.6 \pm 5.6 \mathrm{~kg}$; body height, $174.3 \pm 4.8 \mathrm{~cm}$. The characteristics of the subjects are presented in Table 2. The subjects were randomly assigned either to the SSGTG $(n=10)$ or RSTG $(n=9)$. Goalkeepers were excluded from the study.

The study protocol was approved by the Ethical Committee of the CELAL BAYAR University according to the revised Declaration of Helsinki before the commencement of the study. Written informed consent was received from all players after a brief but detailed explanation of the aims, benefits and risks involved with the process. All players were informed that they could withdraw from the study at any time without consequences.

\section{Anthropometrical Assessments}

The anthropometric measurements included body height, body mass and the sum of measurements taken from 4 skinfold sites with Harpenden callipers (the triceps, subscapular, abdominal, and suprailiac sites) which were used to determine body fat percentages (Yuhasz, 1962).

Yo-Yo Intermittent Recovery Test Level 1

The Yo-Yo IR1 was performed as described by Krustrup et al. (2003). The audio cues for the Yo-Yo IR1 were recorded on a CD (www.teknosport.com, Ancona, Italy). The total distance covered during the Yo-Yo IR1 (including the last incomplete shuttle) was taken as the testing score (Krustrup et al., 2003).

\section{Repeated Shuttle Sprints Ability Test}

This test was an adaptation of a version that had shown to produce reliable and valid estimates of RSA (Impellizzeri et al., 2008). The test was conducted on a natural soccer field and all of the participants wore soccer shoes. The warm-up consisted of $15 \mathrm{~min}$ of low-intensity jogging and dynamic stretching of the lower extremity muscles followed by 2-3 submaximal trial efforts of the test which was about to be conducted. Participants then rested for $5 \mathrm{~min}$ before the start of the RSSAT. The RSSAT involved six repetitions of maximal $2 \times 20 \mathrm{~m}$ shuttle sprints with $20 \mathrm{~s}$ of passive recovery. The players received verbal encouragement during each trial to elicit maximal effort. The sprint time was measured using photocells ( $0.01 \mathrm{~s}$ precision). Repeated shuttle sprint ability was represented by the best sprint time (RSAbestime, in seconds), the mean sprint time (RSAmeantime, in seconds) and the RSA decrement score (RSAdecrement, in percentages). The RSAdecrement (\%) was calculated as follows: 100 - (mean time / best time $\times 100)$ (Rampinini et al., 2007).

\section{Loughborough Soccer Passing Test}

To obtain an objective measure of shortpassing ability, the second version of the LSPT was used. This version had been shown to be both reliable and valid (Ali et al., 2007). Ali et al. (2007) presented the detailed protocol and a schematic representation of the test. The participants performed the test twice and the mean was taken as their performance score. The final LPST performance score was termed the LPST (s) (Ali et al., 2007).

\section{Training Program}

The weekly schedules of the periodized training intervention are shown in the calendar in Table 1 . The first 4 weeks were during the preseason which were parts of the specific preparation training phase and the last 2 weeks were the beginning of the regular season.

The workouts were identical for both groups. Both the SSGT and RST modalities lasted between 60 to $90 \mathrm{~min}$ and were always performed at the beginning of regular training which started with a standardized warm-up. No other endurance, speed-agility and short-passing ability training was completed during the study period.

During the training interventions, the aim for the SSGT group was to train at an intensity corresponding to $90-95 \%$ of $\mathrm{HR}_{\max }$. The individual targeted heart rate during each SSGT unit was controlled and optimized with real-time feedback 
by consistent coach encouragement. Short-range radio telemetry was used during SSGT (Activio Fitness System, Nordenflychtsvagen 66, SE-112 51 Stockholm, Sweden). During the training interventions, the SSGs were based on 3-a-side team format, excluding the goalkeepers, and were conducted on a playing field of a $18 \times 30 \mathrm{~m}$ delimited area. SSGT consisted of 4 sets of $3 \mathrm{~min}$ work periods with $4 \mathrm{~min}$ of passive recovery between sets (Bravo et al., 2008; Hill-Haas et al., 2010; Owen et al., 2004; Rampinini et al., 2007). These game configurations, the size of the game area and the number of players were considered to be appropriate in order to reach the targeted intensity.

On the other hand, the RSTG performed repeated sprints without a ball. Participants were instructed to complete all sprints as fast as possible and strong verbal encouragement was provided to each subject during RST. The RSSAT protocol used by Rampinini et al. (2007) was considered as an appropriate RST method for the current study. RST consisted of 3 sets of $6 \times 40 \mathrm{~m}$ maximal sprints and a passive recovery (Impellizzeri et al., 2008; Rampinini et al., 2007) of $20 \mathrm{~s}$ between the sets and $4 \mathrm{~min}$ between the blocks of sets were allowed. Subjects performed maximal $40 \mathrm{~m}$ straight-line sprints during the first set. Both the second and third sets included maximal $40 \mathrm{~m}$ sprints with two changes of direction, $45^{\circ}$ and $90^{\circ}$, respectively. The distance of all sprints during each repeated-sprint sequence including the changes of direction was equal. The total volume per session was $720 \mathrm{~m}$. Repeated-sprints are intermittent exercises that have a considerable anaerobic contribution and HR can be a poor marker of exercise intensity thus, no HR feedback was provided or recorded.

\section{Game-Specific Skill Involvement}

The small-sided game training was analyzed for the number of game-specific skills performed by the players in order to emphasize the amount of contact with the ball. During the intervention, 2 researchers were assigned to count the number of offensive skills per player (passing, ball control and dribbling).

\section{Statistical analyses}

The statistical package SPSS (16.0) was used for the analysis. Data are expressed as mean \pm standard deviation. The Kolmogorov Smirnov test was first applied to confirm the normal distribution of the data and the Levene's test was applied to assess the homogeneity of variance. Because of the limited number of subjects and lack of homogeneity in groups, Wilcoxon signed Rank and Mann-Whitney $U$ tests were used to examine the differences between groups and training effects.

\section{Results}

There were no significant difference in the baseline anthropometric variables between the groups except for training experience $(p<0.05$, Table 1).

\%HRmean during training sessions of the SSGTG was $89.52 \pm 5.47 \%$ HRmax. The SSGT group performed a total of 5111 offensive actions or skills such as passing, ball control and dribbling during the interventions, while the RSTG did not.

\begin{tabular}{|c|c|c|c|c|c|c|c|}
\hline \multicolumn{8}{|c|}{ Table 1} \\
\hline Week & $\begin{array}{l}\text { Monda } \\
\mathrm{y}\end{array}$ & Tuesday & Wednesday & Thursday & Friday & Saturday & Sunday \\
\hline Pre-test & Rest & LSPT & Rest & Yo-Yo IR1 & Rest & RSSAT & Rest \\
\hline Week 1 & Day off & Str+inter & Agility +Tact & Inter+Tact & Tech & Day off & Fmatch \\
\hline Week 2 & Day off & Str+inter & Agility+Tact & Inter+Tact & Speed+Tech & Day off & Fmatch \\
\hline Week 3 & Day off & Str+inter & Agility+Tact & Inter+Tact & Speed+Tech & Day off & Fmatch \\
\hline Week 4 & Day off & Str+inter & Agility+Tact & Inter+Tact & Speed+Tech & Day off & Fmatch \\
\hline Week 5 & Day off & Str+inter & Agility+Tact & Inter+Tact & Speed+Tech & Day off & Omatch \\
\hline Week 6 & Day off & Str+inter & Agility+Tact & Inter+Tact & Speed+Tech & Day off & Omatch \\
\hline Post-test & Day off & LSPT & Rest & Yo-Yo IR1 & Rest & RSSAT & Rest \\
\hline \multicolumn{8}{|c|}{$\begin{array}{l}\text { Str = strength (low-level prevention sessions); Inter = SSGT } \\
\text { or RSST interventions; Tact = tactical session (low intensity); } \\
\text { al session (goal-scoring), Friendly match = Fmatch; Official match = Omatch }\end{array}$} \\
\hline
\end{tabular}


Table 2

Descriptive characteristics of the subjects.

\begin{tabular}{ccccc}
\hline & $\begin{array}{c}\text { Body } \\
\text { height }(\mathrm{m})\end{array}$ & $\begin{array}{c}\text { Body mass } \\
(\mathrm{kg})\end{array}$ & $\begin{array}{c}\text { Age } \\
(\text { years })\end{array}$ & $\begin{array}{c}\text { Training experience } \\
\text { (years) }\end{array}$ \\
\hline RSTG $\mathrm{n}=9$ & & $65.4 \pm 5.2$ & $16.84 \pm$ & $4.44 \pm 0.88$ \\
SSGTG $\mathrm{n}=10$ & $1.72 \pm 4.82$ & & 1.18 & $5.6 \pm 1.17$ \\
& & $65.8 \pm 5.9$ & $17.07 \pm$ \\
Statistical differences & $1.74 \pm 3.26$ & & 1.22 & $p<0.05$ \\
\hline
\end{tabular}

$p<0.05$, significant difference between the groups; N.S., non-significant difference between the groups.

Table 3

\begin{tabular}{|c|c|c|c|c|c|c|}
\hline \multirow[b]{2}{*}{ Variables } & \multicolumn{3}{|l|}{$\operatorname{RSTG}(\mathrm{n}=9)$} & \multicolumn{3}{|c|}{ SSGTG $(n=10)$} \\
\hline & Pre & Post & Differences & Pre & Post & Differences \\
\hline Body fat (\%) & $10.40 \pm 0.81$ & $10.31 \pm 0.87$ & N.S. & $10.40 \pm 1.21$ & $10.19 \pm 1.16$ & $p=0.05^{*}$ \\
\hline Yo-Yo IR1 (m) & $2306.6 \pm 252.1$ & $2480 \pm 158.7$ & $p=0.010^{* *}$ & $2320 \pm 388$ & $2432 \pm 336$ & N.S. \\
\hline RSAdecrementt $(\%)$ & $5.5 \pm 0.8$ & $4.76 \pm 0.51$ & $p=0.05^{*}$ & $5.80 \pm 1.05$ & $3.75 \pm 1.16$ & $p=0.013^{*}$ \\
\hline RSAmeantime (s) & $7.13 \pm 0.17$ & $7.13 \pm 0.21$ & N.S. & $7.12 \pm 0.17$ & $7.22 \pm 0.20$ & N.S. \\
\hline RSAbesttime (s) & $6.75 \pm 0.19$ & $6.81 \pm 0.18$ & N.S. & $6.73 \pm 0.19$ & $6.96 \pm 0.24$ & $p=0.016^{*}$ \\
\hline LSPT (s) & $36.20 \pm 5.92$ & $37.74 \pm 8.42$ & N.S. & $40 \pm 4.08$ & $33.73 \pm 4.47$ & $p=0.013^{*}$ \\
\hline
\end{tabular}

$*(p \leq 0.05)$ and ${ }^{* *}(p \leq 0.01)$, significant differences between pre and post intervention value.

a)
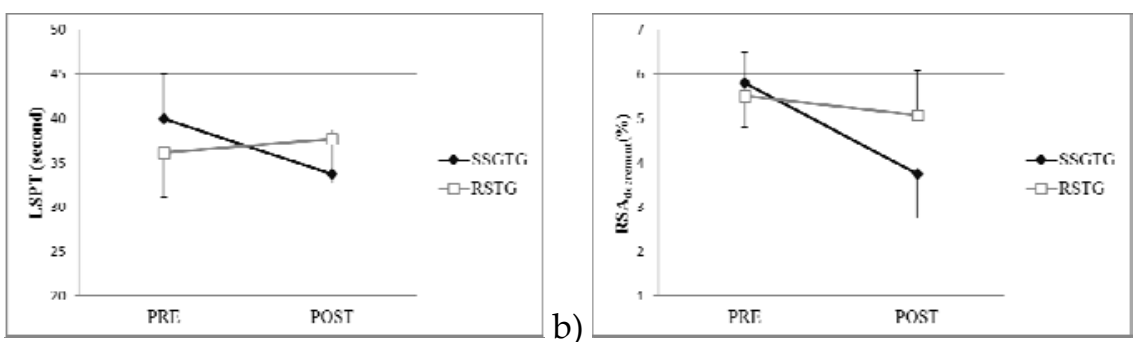

Figure 1a, $\mathbf{b}$

Changes in the LSPT and RSAdecrement performance for SSGTG and RSTG intervention, respectively. 
The SSGT program improved ShortPassing Ability $(p<0.05)$, but had no effect in the RSTG ( $p>0.05$; Table 3 and Figure 1a).

The physical values before and after the interventions are presented in Table 3.

The Yo-Yo IR1 performance levels were improved by the RST program $(p<0.01)$, but there were no improvements in the SSGT group after interventions in terms of the Yo-Yo IR1 performance levels ( $p>0.05$; Table 3$)$.

The RSAdecrement (\%) scores were improved by both the RST and SSGT programs $(p<0.05$, Table 3 and Figure $1 b)$.

\section{Discussion}

The aim of the present study was to investigate the effects of 6-week high intensity SSG and RSA training interventions on RSA performance, soccer specific endurance and short passing ability in elite youth soccer players and to compare the effects of both training modalities.

Effects of RST and SSGT on Yo-Yo IR1 Performance

Results of this study confirm some of the hypotheses. The RS training intervention induced significant improvement in soccer-specific endurance (Yo-Yo IR1), while SSG training did not (Table 3). Hill-Haas et al. (2009) revealed that the Yo-YoIR1 performance was improved by 7 weeks of SSGT (different game formats on various pitch sizes, number of players and duration). Although the Yo-Yo Intermittent Recovery tests have been used extensively in the assessment of soccer-specific endurance of players (Krustrup et al., 2003), there are more measures to analyze the soccer-specific endurance other than the Yo-Yo Intermittent Recovery tests. One of these measures is the Ekblom's circuit test (Balsom, 1994). The lack of significant improvements in soccer specific endurance performance after SSGT intervention in the present study was also confirmed by Impellizzeri (2006) who used the Ekblom's circuit test to measure soccer-specific endurance of players after various SSGT interventions.

In the present study, it seems difficult to determine the mechanisms responsible for the lack of significant improvements in the Yo-Yo IR1 performance in the SSGT intervention group. In some research concerning the related subject, the lack of significant improvements in the Yo-Yo IR1 performance in the SSGT group vs. RST group was linked to the pre-training fitness level within two groups (Bravo et al., 2008). In the current study, the pre-training fitness level of the two groups may not explain this finding since the initial Yo-Yo IR1 performance values were similar. The intensity of SSGT of the present study ( $3 \mathrm{v} \mathrm{s} 3,4 \mathrm{~min}, 89.52 \pm 5.47 \%$ HRmax) might be different than in similar studies, which in return may be the reason for the lack of improvement. Another possible explanation of these conflicting results may be that the individual training intensity differences often lead to lack of improvements in individual performance, which in return may be followed by the lack of significant improvements of the SSGT group in the Yo-Yo IR1 performance. Specifically, these differences occur because of subjects' disobedience to the high intensity training concept. Although constant feedback about the target HR was provided, some subjects might have chosen not to reach the above $90 \%$ HRmax zone.

RST group's improvement in soccerspecific endurance (the Yo-Yo IR1) was consistent with our hypothesis. Similar results after RS training intervention in junior soccer players of a professional team were also found by Bravo et al. ( 2008). A possible explanation of this finding could be related to the physiological requirements of the Yo-Yo IR1, as RS training can induce improvements in both aerobic and anaerobic metabolism which may lead to greater improvement in soccer-specific endurance (as measured by the the Yo-Yo IR1) compared with SSG training (Bravo et al., 2008; Burgomaster et al., 2005; Dawson et al., 1998).

In addition, the RS training protocol included changes of direction (two changes of direction; $45^{\circ}$ and $\left.90^{\circ}\right)$. These direction changes may have positively influenced the performance in the Yo-Yo IR1 test since the test also includes similar direction changes (i.e., shuttle running) (Lakomy and Haydon, 2004).

\section{Effects of RST and SSGT on RSA Performance}

Consistent with the hypothesis, both SSGT and RST had a beneficial impact on the RSAdecrement of the RSSAT, while no change in RSAmeantime was observed contrary to the hypothesis (Table 3 and Figure 1b). The SSG improvement effect was greater than that of the 
RS training program in RSAdecrement (Table 3 and Figure $1 b)$. In the related literature, training studies focusing on the development of RSA in team sport players have reported inconsistent results, since different RSA testing and RST protocols were used (Buchheit et al., 2009; Impellizzeri et al., 2008; Owen et al., 2012). Buchheit et al. (2009) along with Bravo et al. (2008) also noted that studies focused on the development of RSA in team sport players had reported inconsistent results and mostly investigated the impact of dissociated training approaches such as RST, aerobic interval training and aerobic-oriented small-sided games. Inconsistency in the findings of other authors may firstly be related to RSA testing protocols which used various RSA scores for assessment (RSAdecrement, RSAtotal, RSAfatiguetime or RSAmean). Secondly, there are also various ways to interpret the changes in the sprint decrement scores (RSAdecrement). Therefore, it is difficult to achieve conclusive results about the benefits of various repeated-sprint training protocols in RSA comparisons. In a similar study related to RSA performance using a different formula to calculate the RSA percentage decrement score, Owen et al. (2012) showed that the 4 week SSG training (3 vs. $3+$ GKs, $5-11 \times 3 \mathrm{~min}$ duration and a $2 \mathrm{~min}$ passive recovery between bouts) intervention induced significant improvement in RSAdecrement in elite and adult professional soccer players during the in-season break. In a study with different testing and training protocols, Hill-Haas et al. (2009) found a lack of improvement in RSAtotal in both mixed generic fitness training (i.e. aerobic power, prolonged intermittent high intensity running, sprint training (up to a maximum of $20 \mathrm{~m}$ ) and repeatedsprint training) and various SSG training formats (different pitch sizes of $2 \mathrm{v} 2$ to $6 \mathrm{v}$ 6) in soccer players. In another study about SSG training effects on RSA, Jensen et al. (2007) found improvement in fatigue time in a repeated sprint test with different training protocols (30 min SSG with 2-4 min work intervals and 1-2 min of rest) in soccer players.

A study on handball players which is in line with the current study presents similar improvements following both game-based handball training and high-intensity interval training regimens in RSAdecrement, while having different results regarding the improvement of RSAmeantime and RSAbesttime (Buchheit et al., 2009).

\section{Effect of RST on RSA Performance}

In a study inconsistent with the present one, Bravo et al. (2008) observed a decrease in the RSAmeantime, while there was no change in RSAdecrement after RST in soccer players. A study on tennis players resembling Bravo's (2008) research also provided similar results; FernandezFernandez et al. (2012) reported that 3 times per week of 6 weeks, RST led to a significant improvement in the RSAmeantime, but not in RSAdecrement, whereas there were no differences between the pre-test and post-test in the highintensity interval training group. Buchheit et al. (2010) also showed results inconsistent with the present ones indicating that the RS training program (2-3 sets of 5-6 $\times 15$ to $20 \mathrm{~m}$ shuttle sprints interspersed with $14 \mathrm{~s}$ of passive recovery or $23 \mathrm{~s}$ of active recovery) had a likely beneficial impact on RSAbestime and RSAmeantime in the RSA test (6 repetitions of maximal $2 \times 15$ m shuttle sprints ( $\sim 6$ s) departing every $20 \mathrm{~s}$ ) in soccer players.

The last three aforementioned studies indicated no change in RSAdecrement and an improvement in RSAmeantime and RSAbesttime, respectively, unlike the present research. Overall, these studies suggest that RS training elicits enhanced RSAmeantime and RSAbesttime leading to improved anaerobic performance, but does not increase the ability to recover between sprints, which is inconsistent with the present study. This inconsistency may be caused by the difference in the employed testing protocols and training variables.

In other studies related to RSA development, improvement in RSAdecrement through interval training was very likely greater than that resulting from RS training (Mohr et al., 2007; Schneiker and Bishop, 2008). It is possible that the improvement in RSAdecrement could be related to other physiological adaptations that facilitate the recovery process induced by aerobic interval training. The present study shows similar results to the ones presented in the literature related to the effects of high-intensity aerobic interval training on RSAdecrement performance.

With respect to RSA, RST has been reported to produce greater improvements in 
RSAbesttime (Buchheit et al., 2010; Mohr et al., 2007; Schneiker and Bishop, 2008) and RSAmeantime (Buchheit et al., 2010; Bravo et al., 2008; Mohr et al., 2007; Schneiker and Bishop, 2008), compared to interval-based training. Surprisingly, the current study did not provide similar results to the related studies in RSAbestime and RSAmeantime in both training groups; the only improvement observed was in RSAdecrement. Concerning the present research, several studies showed explanatory results and mentioned that RSAdecrement might partially be related to aerobic power, while the improvement in RSAmeantime and RSAbesttime may reflect enhanced anaerobic metabolism, which are also important determinants of RSA and can be increased with sprint training (Burgomaster et al., 2005; Dawson et al., 1998; Jacobs et al., 1987). The present study's RSA and SSG training protocols may provide an adequate stimulus to induce improvement in aerobic fitness which concurrently improves RSAdecrement.

The repeated-sprint training volume (number of sprint or intensity and total distance, recovery time) used in the present study (two sessions a week with 18 shuttle sprints ( $20+20$ m) at maximal intensity) may be similar to or unlike the previous ones. This difference in volume may explain the lack of improvement in RSAmeantime and RSAbesttime and the improvement in RSAdecrement after RSA training which is inconsistent with the findings of previous studies with different volumes of sprintbased training (Bravo et al., 2008; Buchheit et al., 2010; Dawson et al., 1998; Fernandez-Fernandez et al., 2012).

In addition, the fitness level of participants in the current study may cause the difference between the other studies, which makes the results difficult to compare.

As a result, considering various metabolic, neural and mechanical determinants of RSA (Bishop et al., 2011), while different training strategies that target each of these individual components may impact RSA, it is unclear which of them is most effective.

The present study indicates that the improvement in RSAdecrement observed in the SSGTG $(31.15 \%)$ was more evident than that of the RST group (11.56\%). Thus, not only SSG training was more effective, but also its game- based specificity made it more preferential and efficient.

The observed improvement in RSA within the SSGT group is substantial. Considering the time-workloads and intensities, SSGs resemble actual match play, while also developing technical and tactical proficiency, what has led to their popularity as a training modality in the applied and scientific domain within the recent years (Dellal et al., 2011).

Due to the long training time spent with the ball in the SSG sessions, we hypothesized that the SSGT group would present greater improvements in short-passing ability. In agreement with the hypothesis, small-sided games that included a total of 5111 offensive skills (passing, ball control and dribbling) were effective in enhancing short-passing ability performances in elite young soccer players. On the other hand, the present study was the first to demonstrate that high intensity RS training intervention may induce a substantial regression in LSPT results. A $14.7 \%$ improvement in the LSPT was found in the SSGT group, but this rate was only $7.87 \%$ in the RST group. One of the differences between SSG and RS training methods is the presence of the ball which imposes a specific activity and allows the concomitant improvement of technical and tactical skills with high player motivation.

Although the current research used the valid and reliable LSPT (Ali et al., 2007) in order to evaluate short-passing ability, previous studies had researched the effects of SSGT on specific technical soccer skills by using various testing methods, such as ball control (i.e., juggling), passing and dribbling skills. Diverse measurement methods in order to test soccerspecific technical skills may generate different training-induced improvement results that might be the reason for the differences between the current and other studies. A few authors have emphasized the importance of SSGT and interval training which are efficient ways to improve or maintain soccer-specific technical skills (Radziminski et al., 2013; Reilly and White, 2005).

Others studies have produced similar results. Radziminski et al. (2013) showed a significant improvement in the soccer-specific technical skill level in the SSGTG (e.g., 4 x 4 min with $3 \mathrm{~min}$ of active recovery over $90 \%$ of the HRmax) and no improvement in the running 
group after 8 weeks of intervention training. In that study, Radziminski et al. (2013) used the battery of tests proposed by the German soccer federation in order to evaluate the levels of soccerspecific technical skills, such as ball control (i.e., juggling), passing (i.e., against the bench, to the goal, rotation passes, long passes, heading), and dribbling. Another study by Reilly and White (2005) measured soccer dribbling ability, both before and after interval and SSG training. In this study, no deterioration in dribbling ability was observed in either group (Reilly and White, 2005). The difference between the present study and that of Reilly and White's (2005) is that those authors observed no deterioration in dribbling ability, whereas the present study showed improvements in short-passing ability.

Consequently, our results demonstrate that high-intensity SSG training is an effective tool to improve both RSA and technical skills. Highintensity SSG training may be a recommended protocol for soccer players in order to improve both technical skills and RSA simultaneously. Thus, with the addition of SSG training into the regular training protocols, a substantial reduction in exercise training time can be obtained, which in return will allow the players to spend more time on technical-tactical training that will assist in developing their technical and tactical skills.

\section{References}

Ali A, Williams C, Hulse M, Strudwick A, Reddin J, Howarth L, Eldred J, Hirst M, McGregor S. Reliability and validity of two tests of soccer skill. J Sport Sci, 2007; 25(13): 1461-1470

Aziz A, Chia M, Teh K. The relationship between maximal oxygen uptake and repeated sprint performance indices in field hockey and soccer players. J Sport Med Phys Fit, 2000; 40: 195-200

Aziz A, Mukherjee S, Chia M, Teh K. Validity of the running repeated sprint ability test among playing positions and level of competitiveness in trained soccer players. Int J Sports Med, 2008; 29: 833-838

Balsom P. Evaluation of physical performance. In B. Ekblom (Ed.), Football (Soccer) (pp. 102-123). Oxford, UK: Blackwell Scientific Publications; 1994

Bangsbo J. The physiology of soccer with special reference to intense intermittent exercise. Acta Physiol Scand (Supplementum), 1994; 619: 1-155

Bangsbo J, Iaia FM, Krustrup P. The Yo-Yo Intermittent Recovery Test. Sports Med, 2012; 38: 37-51

Bishop D, Girard O, Mendez-Villanueva A. Repeated-sprint ability part II: Recommendations for training. Sports Med, 2011; 41: 741-756

Bravo DF, Impellizzeri FM, Rampinini E, Castagna C, Bishop D, Wisloff U. Sprint vs. Interval Training in Football. Int J Sports Med, 2008; 29: 668-674

Buchheit M, Laursen PB, Kuhnle J, Ruch D, Renaud C, Ahmaidi S. Game-based training in young elite handball players. Int J Sports Med, 2009; 30: 251-258

Buchheit M, Mendez A, Delhomel G, Brughelli M, Ahmaidi S. Improving repeated sprint ability in young elite soccer players: repeated shuttle sprints vs. explosive strength training. J Strength Cond Res, 2010; 24: $2715-2722$

Burgomaster KA, Hughes SC, Heigenhauser GJF, Bradwell SN, Gibala MJ. Six sessions of sprint interval training increases muscle oxidative potential and cycle endurance capacity in humans. J Appl Physiol, 2005; 98: 1985-1990

Chamari K, Hachana Y, Kaouech F, Jeddi R, Moussa-Chamari I, Wisløff U. Endurance training and testing with the ball in young elite soccer players. Brit J Sport Med, 2005; 39: 24-8

Dawson B, Fitzsimons M, Green S, Goodman C, Carey M, Cole K. Changes in performance, muscle metabolites, enzymes and fibre types after short sprint training. Eur J Appl Physiol O, 1998; 78: 163-169

Dellal A, Hill-Haas S, Lago-Penas C, Chamari K. Small-sided games in soccer: amateur vs. professional players' physiological responses, physical, and technical activities. J Strength Cond Res, 2011; 25: 23712381

Edge J, Bishop D, Goodman C, Dawson B. Effects of high- and moderate-intensity training on metabolism and repeated sprints. Med Sci Sport Exer, 2005; 37: 1975-1982 
Fernandez-Fernandez J, Zimek R, Wiewelhove T, Ferrauti A. High-Intensity Interval Training vs. RepeatedSprint Training in Tennis. J Strength Cond Res, 2012; 26: 53-62

Ferrari Bravo D, Impellizzeri FM, Rampinini E, Castagna C, Bishop D, Wisloff U. Sprint vs. interval training in football. Int J Sports Med, 2008; 29: 668-674

Gibala MJ, Little JP, Van Essen M, Wilkin GP, Burgomaster KA, Safdar A, Raha S, Tarnopolsky MA. Shortterm sprint interval versus traditional endurance training: similar initial adaptations in human skeletal muscle and exercise performance. J Physiol, 2006; 575: 901-911

Hill-Haas SV, Coutts AJ, Dawson BT, Rowsell GJ. Time-motion characteristics and physiological responses of small-sided games in elite youth players:the influence of player number and rule changes. J Strength Cond Res, 2010; 24: 2149-56

Hill-Haas SV, Coutts AJ, Rowsell GJ, Dawson BT. Generic versus small-sided game training in soccer. Int J Sports Med, 2009; 30: 636-642

Hughes M, Franks I. Analysis of passing sequences, shots and goals in soccer. J Sport Sci, 2005; 23: 509-514

Impellizzeri FM, Marcora SM, Castagna C, Reilly T, Sassi A, Iaia FM, Rampinini E. Physiological and performance effects of generic versus specific aerobic training in soccer players. Int J Sports Med, 2006; 27: 483-492

Impellizzeri FM, Rampinini E, Castagna C, Bishop D, Ferrari Bravo D, Tibaudi A, Wisloff U. Validity of a repeated-sprint test for football. Int J Sports Med, 2008; 29: 899-905

Jacobs I, Esbjörnsson M, Sylvén C, Holm I, Jansson E. Sprint training effects on muscle myoglobin, enzymes, fiber types, and blood lactate. Med Sci Sport Exer, 1987; 19: 368-374

Jensen J, Randers M, Krustrup P, Bangsbo J. Effect of additional in-season aerobic high-intensity drills on physical fitness of elite football players. J Sports Sci Med ((Supplementum), 2007; 79

Katis A, Kellis E. Effects of small-sided games on physical conditioning and performance in young soccer players. J Sports Sci Med, 2009; 8: 374-380

Krustrup P, Mohr M, Amstrup T, Rysgaard T, Johansen J, Steensberg A, Pedersen PK. The Yo-Yo intermittent recovery test: Physiological response, reliability, and validity. Med Sci Sport Exer, 2003; 35: 697-705

Lakomy J, Haydon DT. The effects of enforced, rapid deceleration on performance in a multiple sprint test. J Strength Cond Res, 2004; 18: 579-583

Mohr M, Krustrup P, Nielsen JJ, Nybo L, Rasmussen MK, Juel C, Bangsbo J. Effect of two different intense training regimens on skeletal muscle ion transport proteins and fatigue development. Am J Physiol-Reg I, 2007; 292: R1594-602

Owen AL, Wong DP, Paul D, Dellal A. Effects of a periodized small-sided game training intervention on physical performance in elite professional soccer. J Strength Cond Res, 2012; 26: 2748-54

Owen A, Twist C, Ford P. Small-Sided Games: the Physiological and Technical Effect of Altering Pitch Size and Player Numbers. Insight, 2004; 7: 50-53

Radziminski L, Rompa P, Barnat W, Dargiewicz R, Jastrzebski Z. A Comparison of the Physiological and Technical Effects of High-Intensity Running and Small-Sided Games in Young Soccer Players. Int J Sports Sci Coach, 2013; 8: 455-465

Rampinini E, Bishop D, Marcora SM, Ferrari Bravo D, Sassi R, Impellizzeri FM. Validity of simple field tests as indicators of match-related physical performance in top-level professional soccer players. Int J Sports Med, 2007; 28: 228-235

Rampinini E, Impellizzeri FM, Castagna C, Abt G, Chamari K, Sassi A, Marcora SM. Factors influencing physiological responses to small-sided soccer games. J Sports Sci, 2007; 25: 659-666

Rampinini E, Sassi A, Morelli A, Mazzoni S, Fanchini M, Coutts AJ. Repeated-sprint ability in professional and amateur soccer players. Appl Physiol Nutr Metab, 2009; 34: 1048-1054

Reilly T, White C. Small-sided games as an alternative to interval-training for soccer players. In T. Reilly, J. Cabri, D. Araújo (Ed.), Science and Football V. (pp. 344-347). London and New York: Routledge; 2005

Reilly T, Gilbourne D. Science and football: a review of applied research in the football codes. J Sports Sci, 2003; 21: 693-705

Reilly T, Williams AM, Nevill A, Franks A. A multidisciplinary approach to talent identification in soccer. J 
Sports Sci, 2000; 18: 695-702

Sajadi N, Rahnama N. Analysis of goals in 2006 FIFA World Cup. J Sports Sci Med, 2007; 6: (Suppl.10) 3

Schneiker KT, Bishop D. The effects oh high-intensity interval training vs intermittent sprint training on physiological capacities important for team sport performance. In: Burnett A, (Ed.) Science and nutrition in exercise and sport. Melbourne (VIC): Exerc Sport Sci Aust; 2008

Serpell BG, Young WB, Ford M. Are the perceptual and decision-making components of agility trainable? A preliminary investigation. J Strength Cond Res, 2011; 25: 1240-1248

Spencer M, Bishop D, Dawson B, Goodman C. Physiological and metabolic responses of repeated-sprint activities: specific to field-based team sports. Sports Med, 2005; 35: 1025-1044

Wisloff U. Strong correlation of maximal squat strength with sprint performance and vertical jump height in elite soccer players. Brit J Sport Med, 2004; 38: 285-288

Yuhasz M. The effects of sports training on body fat in man with predictions of optimal body weight. Urbana, University of Illinois; 1962

\section{Corresponding author:}

\section{Niyazi Eniseler,}

Department of Physical Education and Sports, Celal Bayar University, Şehzadeler, 45010, Manisa, Turkey. Phone: 905334679975

Fax: 902362313001

E-mail : niyazieniseler66@gmail.com 